\title{
ANÁLISE DE IMAGENS PARA A CARACTERIZAÇÃO DAS ATIVIDADES DE VACAS LEITEIRAS DENTRO DO GALPÃO DE CONFINAMENTO
}

\author{
SILVIA R. L. DE SOUZA ${ }^{1}$, IRENILZA DE A. NÄÄS ${ }^{2}$, DANIELLA J. DE MOURA ${ }^{3}$
}

\begin{abstract}
RESUMO: A produção atual de animais necessita do emprego de tecnologias de informação automatizadas, inerentes ao ambiente em que os animais estão inseridos, para que se possa analisar a interferência dos mesmos na produção e no bem-estar. O objetivo desta pesquisa foi comparar a eficiência de duas metodologias de análise de imagens, utilizadas para avaliar a presença de vacas leiteiras em locais específicos no galpão de confinamento. O experimento foi realizado em uma fazenda comercial, e as vacas foram monitoradas através de seis microcâmeras. Foram usadas duas formas de análise da presença das vacas nos locais pré-selecionados (camas, bebedouros e comedouros): imagens analisadas visualmente da tela do computador (T1) e automaticamente, pelo software desenvolvido para a captura de imagens das vacas no galpão de freestall (T2). Os dados de presença foram analisados utilizando a técnica de análise de componentes principais e, para as comparações dos dois métodos, aplicou-se o Teste $\mathrm{t}$ - Student, com 95\% de confiabilidade, para as médias da contagem da presença das vacas em cada área estudada. Não foi encontrada diferença significativa entre os métodos, sendo ambos eficientes para o registro das vacas em cada atividade.
\end{abstract}

PALAVRAS-CHAVE: comportamento, análise de imagens, zootecnia de precisão.

\section{IMAGE ANALYSIS FOR CHARACTERIZATION THE ACTIVITIES OF DAIRY COWS INSIDE THE CONFINEMENT HOUSING}

\begin{abstract}
Today's animal production need the use of information technology and automation in the animal's rearing environment in order to analyze their interference both in the production and animal welfare. The objective of this research was to compare the efficiency of two methodologies of image analysis for evaluating the presence of dairy cows in specific places in the confinement shed. The experiment was done in a commercial farm and the cows were monitored by six cameras. Two ways of analyzing the presence of the cows in the pre- selected places (bedding, drinker and feeder) were used: images analyzed as seen in the computer screen (T1), and automatically by the software developed for capturing the images of the cows in the freestall shed (T2). The attendance data were analyzed using the technique of the principal component analysis and to compare the two methods, it was applied the Student $\mathrm{t}$ - test with $95 \%$ reliability for the mean count of the presence of cows in each studied area. No significant difference between the methods was found, and both methods were efficient for registering the presence of cows in the activities.
\end{abstract}

KEYWORDS: behavior, image analysis, precision livestock production.

\section{INTRODUÇÃO}

O desempenho de vacas leiteiras está associado à nutrição e sanidade, ao sistema de produção e bem-estar do animal, bem como a fatores externos e à interação com tratadores (BOKKERS, 2006). O comportamento é a resposta que o animal apresenta através das condições de manejo e alojamento aos quais estão inseridos, e sua análise poderá contribuir para a otimização do sistema de produção, sendo a observação visual a forma mais simples do registro de determinado comportamento (SARIEGO, 2005). A tarefa de observar e registrar o comportamento animal, de forma contínua, é cansativa e subjetiva (NOLDUS et al., 2000) e pode ser efetuada usando pelo

\footnotetext{
${ }^{1}$ Zootecnista, Doutora em Engenharia Agrícola, FEAGRI-UNICAMP, silucas_souza@ hotmail.com.

${ }^{2}$ Eng ${ }^{a}$ Civil, Professora Colaboradora, FEAGRI-UNICAMP, irenilza@ feagri.unicamp.br.

${ }^{3}$ Eng ${ }^{\mathrm{a}}$ Agrônoma, Professora Doutora, FEAGRI-UNICAMP, daniella.moura@ feagri.unicamp.br.

Recebido pelo Conselho Editorial em: 12-8-2010

Aprovado pelo Conselho Editorial em: 15-8-2011
} 
menos três tipos de formas de visualização: 1- visual direto, em que o avaliador está presente no ambiente do animal (TREVISAN et al., 2004); 2 - visual indireto, em que o avaliador registra os dados de comportamento de maneira automática e analisa-os através de observação visual (BARBOSA FILHO et al., 2007; PEREIRA et al., 2005), e 3 - visão computacional, em que um software processa o armazenamento de imagens em um banco de dados e interpreta-as, de acordo com um algoritmo desenvolvido, podendo fornecer ao usuário um diagnóstico, sem a interferência do avaliador (PALMER \& WAGNER-STORCH, 2003).

O objetivo deste trabalho foi comparar dois métodos de análise de imagens, visual indireto e usando visão computacional, para avaliar a atividade de vacas leiteiras dentro do galpão de confinamento, dos seguintes locais: cama, bebedouro e comedouro, em função da temperatura ambiente.

\section{MATERIAIS E MÉTODOS}

$\mathrm{O}$ experimento foi conduzido em um rebanho leiteiro comercial, alojado em sistema de freestall, no Estado de São Paulo (latitude de $22^{\circ} 32^{\prime}$ '55" sul, longitude de 47 $54^{\prime}$ '50" oeste e altitude de 580 m), com clima Cwa (classificação Köeppen), sendo quente e úmido, com estação chuvosa no verão e seco no inverno.

Animais, alojamento e manejo: Foram selecionadas 80 vacas de um lote com características homogêneas (pluríparas em lactação, peso médio de $600 \mathrm{~kg}$ ) da raça Holandesa (puras por cruza) e em fase de lactação semelhante (entre 90 - 100 dias), com uma produção média de $30 \mathrm{~kg}$ de leite $\mathrm{dia}^{-1}$. O galpão freestall, com área de $80 \mathrm{~m}^{2}$, tinha ventiladores $(\varphi=0,9 \mathrm{~m}$, a cada $11,0 \mathrm{~m}$, com velocidade média do ar $=2,5 \mathrm{~m} \mathrm{~s}^{-1}$ ) e aspersores, com espaçamento entre bicos de 1,0 $\mathrm{m}$ (acionados por uma bomba com vazão da água na linha de aspersão de $30 \mathrm{~L} \mathrm{~h}^{-1}$ e a intermitência de 12 min), instalados nas áreas de comedouro e camas. Na área de alimentação, havia oito ventiladores instalados a 2,5 $\mathrm{m}$ de altura e quatro, na área das camas, a 2,0 $\mathrm{m}$ de altura. Os ventiladores eram acionados usando termostato, quando a temperatura ambiente atingia $25^{\circ} \mathrm{C}$. As vacas eram ordenhadas três vezes ao dia (1h00min, 9h00min e 17h00min), e suas produções, registradas. Os horários de arraçoamento ( $7 \mathrm{~h} 00 \mathrm{~min}$ e $15 \mathrm{~h} 30 \mathrm{~min})$ e a dieta alimentar foram mantidos de acordo com a rotina da fazenda.

Tratamentos: O registro da atividade das vacas nos locais de estudo (cama, bebedouro e comedouro) foi efetuado por duas formas: visual indireto (T1), em que as imagens foram registradas e a contagem das vacas presentes nos locais foi feita visualmente na tela do computador, e através da visão computacional (T2), em que as imagens foram registradas, e a contagem, feita automaticamente, pelo software desenvolvido para a captura de imagens das vacas presentes nos locais.

Procedimento experimental: O registro automático da presença na cama e no comedouro e bebedouro foi feito mediante a instalação de um sistema de captura de imagens, composto por seis microcâmeras coloridas (36 mm x $36 \mathrm{~mm}$ ) analógicas, de 420 linhas horizontais de resolução, com sensibilidade de $11 \mathrm{x}, 12 \mathrm{~V}$ de tensão e $120 \mathrm{~mA}$, com lente convergente de 3,6 m. As câmeras com as lentes de 120 graus foram instaladas no interior do galpão, a $5 \mathrm{~m}$ de altura, e ajustadas com um ângulo de forma a obter a melhor imagem possível. Foram instaladas, na posição de topo, duas câmeras na região das camas (fixadas na estrutura metálica do telhado), três câmeras na zona de alcance dos comedouros (fixadas nos pilares de frente aos comedouros) e duas direcionadas para o bebedouro central (fixadas na estrutura metálica do telhado), e a área em vermelho foi a área de alcance das videocâmeras, conforme o esquema da Figura 1. 


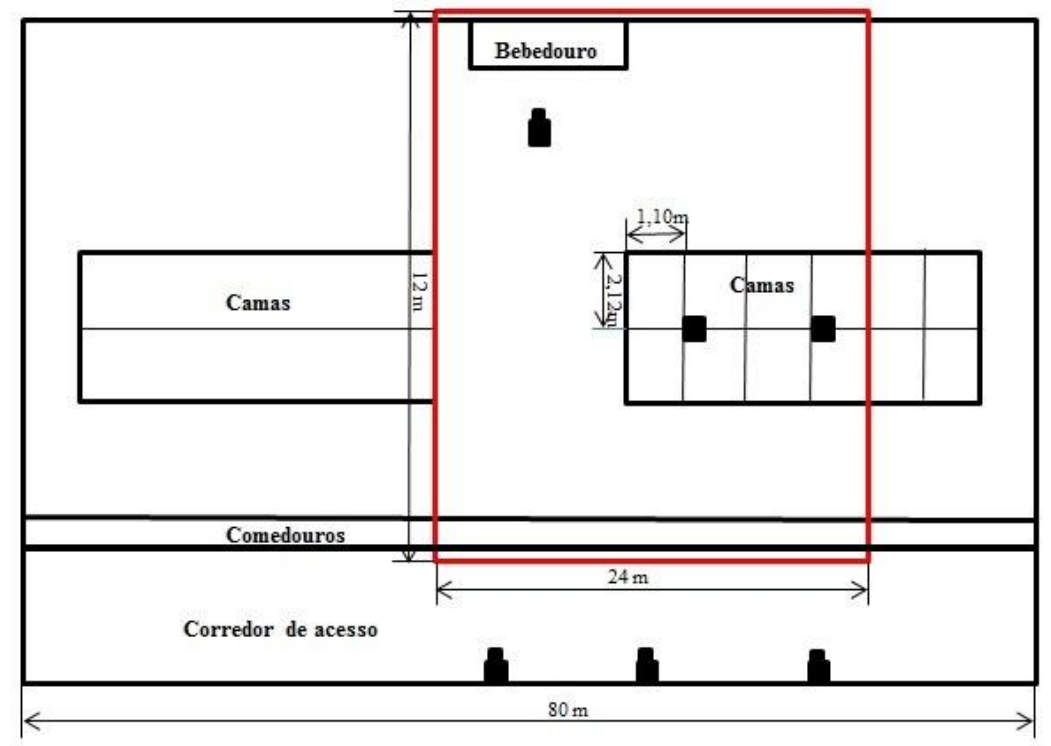

Video Câmeras

FIGURA 1. Esquema da localização das microcâmeras no galpão de freestall. Scheme of the micro cameras localization inside the freestall shed.

Desenvolvimento do programa computacional: Um software foi desenvolvido em Delphi ${ }^{\circledR}$, para o armazenamento de dados e a leitura eletrônica contínua. Um computador Intel Pentium 4 (3,0 Ghz, 1,0 Gb memória DDR 400 (2 x 512 Mb), HD 80 Gb SATA 1507200 rpm Seagate, placa de rede 10/100 Mbps; placa VGA 64 Mb Geforce 4 MX 4000 AGP) foi usado para registro e processamento dos dados. Um equipamento de interface foi desenvolvido para controlar a captura das imagens e o processamento dos dados. A interface entre as câmeras e o computador foi constituída por controladores baseados em seis relés e um driver (ULN2003, ST microeletrônica), com função de controlar os sinais de vídeo de entrada das câmeras via software. Foram registradas a frequência e a quantidade de animais que estavam presentes nos locais estudados. Foi anotado o momento de registro (dia, hora e minuto), e estas imagens foram armazenadas em pastas, organizadas por dia de registro.

O painel de controle do software tinha as seguintes funções: 1 - habilitar o usuário à escolha da porta para a coleta das imagens; 2 - definir o tempo de coleta de cada ciclo das câmeras, e 3 definir o intervalo de tempo entre a imagem coletada por uma câmera e a câmera seguinte. Este intervalo de tempo foi superior $0,5 \mathrm{~s}$, para atender à demanda da resposta do relé (suficiente para transportar o registro feito pelas câmeras). As imagens foram armazenadas pixel a pixel, e a leitura dos pixels foi feita linha por linha, até se obter a imagem completa. Quando se localizava um pixel dentro dos níveis de cores desejados e preestabelecidos, este era armazenado em uma tabela, com sua posição geométrica referenciada (x,y) e níveis de cores registrados em bytes. O processamento do software permitiu adicionar valores de cada componente de RGB (Red, Green e Blue), em números inteiros entre 0 e 255.

Após o reconhecimento de todos os pontos, o uso do software permitia calcular o centro de massa $(\mathrm{CM})$ da figura do animal e a distância de cada pixel até este centro de massa $\left(\mathrm{d}_{\mathrm{i}}\right)$, como mostram, respectivamente, as Equações 1 e 2.

$$
\mathrm{CM}=\left(\frac{\sum d_{x}}{n}, \frac{\sum d_{y}}{n}\right)
$$

em que se atribuiu 1 ao valor de massa de cada pixel. A dispersão indicou a distância média dos pixels até o centro de massa. A dispersão é importante para se definir a aglomeração dos animais. 
Quanto menor o valor de dispersão maior será a aglomeração dos animais do centro de massa. A dispersão foi calculada por:

$$
\mathrm{D}_{\mathrm{i}}=\sqrt{\left(\mathrm{CM}_{\mathrm{x}}-X_{i}\right)^{2}+\left(\mathrm{CM}_{\mathrm{y}}-Y_{i}\right)^{2}}
$$

em que $\mathrm{X}_{\mathrm{i}}$ e $\mathrm{Y}_{\mathrm{i}}$ são as coordenadas do pixel i. Com esses dados, é possível obter outras informações de interesse, como a velocidade média de deslocamento do centro de massa da figura $\left(\overline{\mathbf{V}}_{\mathrm{cm}}\right)$, a dispersão ( Disp ) e a velocidade média de dispersão $\left(\overline{\mathbf{V}}_{\text {disp }}\right)$ dos pontos da figura, como pode ser observado, respectivamente, pelas eq.(3), (4) e (5):

$$
\begin{aligned}
& \overline{\mathbf{V}}_{\mathrm{cm}}=\frac{\Delta d_{c m}}{\Delta \mathrm{t}} \\
& \text { Disp }=\frac{\sum D_{i}}{n} \\
& \overline{\mathbf{V}}_{\mathrm{disp}}=\frac{\Delta D i s p}{\Delta \mathrm{t}}
\end{aligned}
$$

Registro de Atividade: Corrigida a luminosidade do galpão e consultados os tratadores, para melhor entendimento da movimentação dos animais em certos horários (7h00 min e 15h30min), foram feitos vídeos e procedeu-se com à observação visual indireta das vacas, durante $10 \mathrm{~min}$, a fim de se estabelecer um padrão de ocupação, considerado controle, por três dias consecutivos, de maneira a subsidiar a análise (ROUSING \& WEMELSFELDER, 2006; MÜLLER \& SCHRADER, 2005). O processamento das imagens permitiu registrar a movimentação do centro de gravidade dos animais, além do período de tempo destes em cada atividade.

Para o registro de $\mathrm{T}$, foram realizadas observações a cada 10 min durante o dia, enquanto as observações de T2 foram programadas e sincronizadas para registrar $10 \mathrm{~min}$, no mesmo período em que foram programados os dataloggers $\left(\mathrm{HOBO}^{\circledR}\right.$, altura de $2,5 \mathrm{~m}$ ) para os registros das temperaturas mínimas (07h00min) e máximas do dia (15h30min).

Análise Estatística: Utilizou-se a técnica de análise de componentes principais, que apresenta as correlações por meio de representações vetoriais de cada variável estudada, bem como suas intensidades. As correlações ou associações foram analisadas de acordo com a magnitude dos vetores e sua direção. Com essa técnica, foram analisados os dados de presença nos locais predeterminados, em função dos dados de ambiência e verificada a associação de determinada atividade (deitar, beber e comer) em local específico, com a temperatura ambiente. Para a comparação dos dois métodos de análises de imagens, utilizou-se o teste $\mathrm{t}$ - Student, com confiabilidade de 95\%. Foram observadas as quantidades de vacas presentes na cena, quando estavam deitadas na cama, bebendo água ou comendo. Para o processamento de dados, utilizou-se o software estatístico MINITAB (2004).

\section{RESULTADOS E DISCUSSÃO}

A trajetória da vaca observada foi considerada como a projeção de um objeto $\mathrm{V}$ em movimento no plano da imagem $(x, y)$. O registro de sua localização $i$, em determinado instante $t$, era determinado como $(x i, y i)$ em $(x, y, t)$ para $t=$ ti. A trajetória da vaca $T(V)$ foi definida pela sequência do ponto:

$$
\mathrm{T}(\mathrm{V})=\{(\mathrm{x} 1, \mathrm{y} 1, \mathrm{t} 1),(\mathrm{x} 2, \mathrm{y} 2, \mathrm{t} 2), \ldots,(\mathrm{xn}, \mathrm{yn}, \mathrm{tn})\}
$$

em que n é o comprimento da sequência. Dessa forma, a trajetória total foi uma série de deslocamentos em determinada direção e pôde ser representada como t variando de 0 a n, em que $n$ 
foi o tempo de observação $(n=10$ min em várias sequências, para T1 e $n=9$ horas seguidas, para T2). O vetor direcional determinou o deslocamento da vaca, saindo de um ponto e chegando a outro.

Atividades das vacas através do método visual indireto (T1): Foi encontrada pouca associação entre a atividade de beber e a temperatura ambiente $(\mathrm{P}>0,05)$, não tendo sido detectada correlação com as demais atividades (Figura 2).

Como o movimento da vaca é lento (MÜLLER \& SCHRADER, 2005), provavelmente o tempo de 10 min de observação não foi suficiente para a detecção das atividades inerentes aos comportamentos estudados, diferente do recomendado por O'CONNELL et al. (2010) e SCHUTZ et al. (2009).

T1

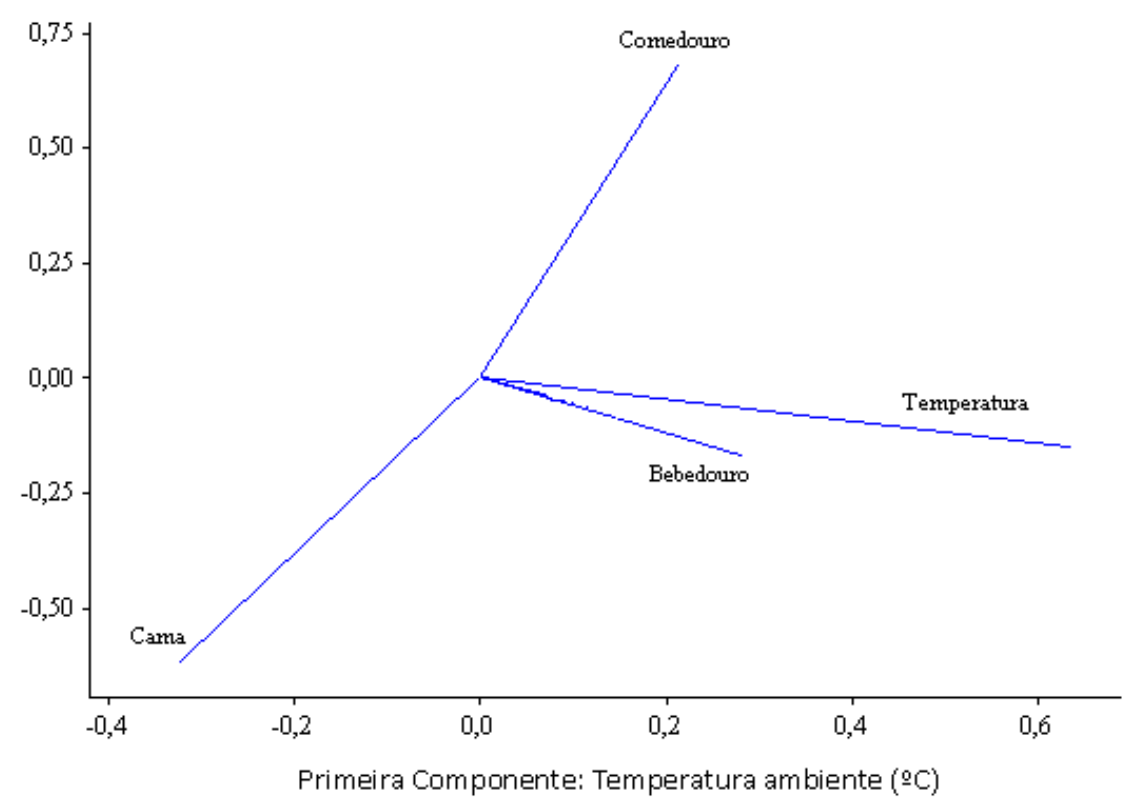

FIGURA 2. Gráfico da análise de componentes principais indicando as preferências de ida das vacas aos locais de estudo, utilizando análise visual indireta e sua associação com a temperatura ambiente. Graph of the principal components analysis indicating the cows preference to the studied places, using indirect visual analyzes and its association with ambient temperature.

A frequência de utilização do comedouro foi inversamente associada com a utilização da cama $(\mathrm{P}<0,05)$, resultado da escolha pelos animais (COSTA \& BROOM, 2001). Não houve correlação positiva entre temperatura ambiente e utilização da cama $(P=0,06)$, embora se estimasse que as temperaturas mais amenas nesta área incentivassem a presença das vacas na cama, conforme encontraram PERISSINOTTO et al. (2009).

Comportamento utilizando visão computacional (T2): Poucos foram os animais que estiveram presentes nas imagens, devido ao alcance limitado das lentes e, algumas vezes, a câmera não conseguiu registrá-los. A análise de componentes principais (Figura 3) indicou pouca associação positiva entre a utilização do bebedouro e a temperatura ambiente, confirmando a tendência de que as vacas bebem mais água, quando a temperatura ambiente aumenta (TEIXEIRA et al., 2006). 


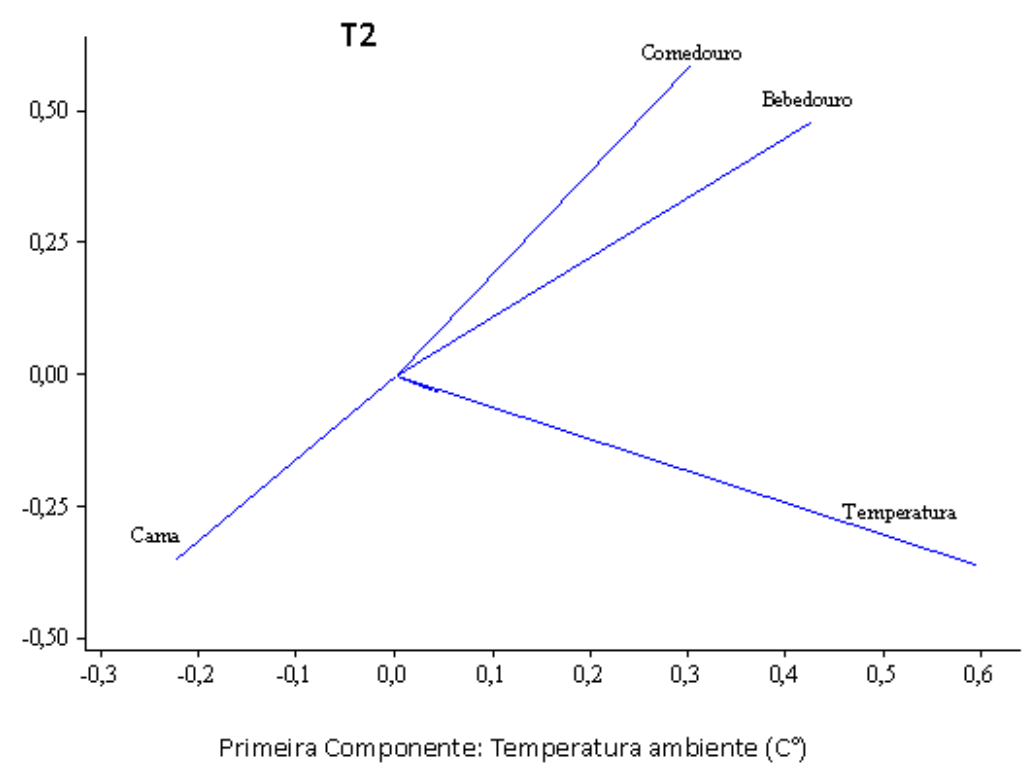

FIGURA 3. Gráfico de componentes principais indicando as preferências de ida das vacas aos locais de estudo, utilizando os dados provenientes da visão computacional e sua associação com a temperatura ambiente. Graph of the principal components analysis indicating the cows preference to the studied places using the data from the computational vision and its association with the ambient temperature.

Observou-se pouca associação negativa entre a atividade de comer e a temperatura ambiente, indicando que as vacas tendem a diminuir a presença no comedouro, quando a temperatura ambiente aumenta. Resultado similar foi descrito por FRAZZI et al. (2000), que encontraram que a distribuição espacial dos animais, dentro do galpão de freestall, reflete sua preferência, em função de variáveis do ambiente interno.

Comparação entre os dois métodos de análise de imagens T1 e T2: Foram realizadas três contagens de vacas presentes em cada local (cama, bebedouro e comedouro). As médias usando T2 não diferiram de T1 $(\mathrm{P}=0,07)$. Entretanto, somente nove vacas estiveram presentes na contagem simultânea, levando a um desvio-padrão alto. Caso o número de vacas tivesse sido maior, o efeito da contagem poderia ter alcançado o nível de significância (Tabela 1).

TABELA 1. Contagem de presença de vacas nos locais predeterminados, para os dois métodos de análise de imagens. Counting of the presence of cows in the predetermined sites using two methods of image analysis.

\begin{tabular}{cccccc}
\hline & T1 & dp & T2 & dp & P-valor \\
\hline Cama & & & & & \\
07h00min & 6 & 5 & 4 & 2 & $>0,05$ \\
15h30min & 4 & 3 & 4 & 3 & $>0,05$ \\
\hline Bebedouro & 2 & 2 & 4 & 2 & $=0,07$ \\
07h00 min & 4 & 2 & 5 & 2 & $=0,07$ \\
15h30min & 2 & 3 & 1 & 1 & $>0,05$ \\
\hline Comedouro & 2 & 1 & 2 & 1 & $>0,05$ \\
07h00min & & & & &
\end{tabular}

Em T2 apresentou-se uma diferença com relação à quantidade de vacas presentes na cena; em relação a $\mathrm{T}$ 1, porém, essa diferença não foi significativa $(\mathrm{P}>0,05)$. Essa diferença pode ser 
explicada pelo tempo de ajuste do início de contagem, adotado pelo algoritmo. A contagem de um animal presente no local não apresentava problemas no registro individual, mas, ocorrendo o agrupamento, o software eventualmente registrava uma única massa, dificultando a determinação da quantidade real de animais agrupados. Atribuiu-se isto às manchas da pelagem das vacas se confundirem durante o processamento das imagens. Vários autores (SCHWAGER et al., 2007; CANGARA et al., 2008) indicam que a automação do processo de monitoramento de animais pode representar um avanço tecnológico considerável. Entretanto, os autores recomendam que se aprofundem mais os estudos nesta área. Este presente trabalho, usando o atual estágio tecnológico, não encontrou resultados que justifiquem a completa automação do processo de monitoramento de atividades em vacas leiteiras, utilizando análise de imagens.

\section{CONCLUSÕES}

Não houve diferença na análise das atividades de vacas leiteiras alojadas em freestall, utilizando dois métodos distintos de observação visual: visão indireta e visão computacional. É necessário maior aperfeiçoamento do algoritmo computacional, para que haja mais eficiência na automação completa do processamento das imagens.

\section{AGRADECIMENTOS}

À FAPESP, pela concessão da bolsa de doutorado, e à FEAGRI/UNICAMP, pelo apoio à pesquisa.

\section{REFERÊNCIAS}

BARBOSA FILHO, J.A.D.; SILVA, I..J.O.; SILVA, M.A.N.; SILVA, C.J.M. Avaliação dos comportamentos de aves poedeiras utilizando sequência de imagens. Engenharia Agrícola, Jaboticabal, v.27, n.1, p.93-99, 2007. Disponivel em: http://www.scielo.br/scielo.php?script=sci_arttext\&pid=S010069162007000100002\&lng=en\&nrm=iso. Acesso em: 7 ago. 2010.

BOKKERS, E.A.M. Effects of interaction between humans and domesticated animals. In: HASSINK, J.; VANDIJK, M. Farming for health. Wageningen: Wageningen University and Research Centre, 2006. cap 3, p.31-41.

CANGARA, O.; LEROYAEROYA, T.; GUARINOB ,M.; VRANKENA ,E.; FALLONC, R.; LENEHANC, J.; MEED, J.; BERCKMANSA, D. Automatic real-time monitoring of locomotion and posture behaviour of pregnant cows prior o calving using online image analysis. Computers and Electronics in Agriculture, Amsterdam, v.64, p.53-60, 2008.

COSTA, M.J.R.; BROOM, D.M. Consistency of side choice in the milking parlour by HolsteinFriesian cows and its relationship with their reactivity and milk yield. Applied Animal Behaviour Science, Bristol, v.70, n.3. p.177-186, 2001.

FRAZZI, E.; CALAMARI, L.; CALEGARI, F.; STEFANINI, L. Behavior of dairy cows in response to different barn cooling systems. Transactions of the American Society of Agricultural Engineers, St. Joseph, v.43, n.2, p.387-394, 2000.

MINITAB. Statistical Software, version 14. 2004. Cd-ROM.

MÜLLER, R.; SCHRADER, L. A. new method to measure behavioral activity levels in dairy cows. Applied Animal Behaviour Science, Bristol, v.83, n.4, p.247-258, 2005.

NOLDUS, L.P.J.J.; TRIENES, R.J.H.; HENDRIKSEN, A.H.; JANSEN, H.; JANSEN, R.G. The Observer Video-Pro: New software for the collection, management, and presentation of timestructured data from videotapes and digital media files. Behavior Research Methods, Instruments and Computers, Amsterdam, v.32, n.1, p.197-206, 2000. 
O'CONNELL, N. E.; FERRIS, C. P.; PATTERSON, D.C.; MAYNE, C.S. Effect of feed barrier design and feed space allowance on performance and behavioural parameters in dairy cows. Applied Animal Behaviour Science, Bristol, v.127, p.20-27, 2010.

PALMER, R.W.; WAGNER-STORCH, A.M. Cow preference for different freestall bases in pens with different stocking rates. In: INTERNATIONAL DAIRY HOUSING, 5., Fort Worth.

Proceedings... Fort Worth: American Society of Agricultural Engineers, 2003. p.155-164.

PEREIRA, D.F.; NÄÄS, I.A.; ROMANINI, C.B.; SALGADO, D.D.; PEREIRA, G.O.T.

Indicadores de bem-estar baseados em reações comportamentais de matrizes pesadas. Engenharia Agrícola, Jaboticabal, v.25, n.2, p.308-314, 2005. Disponivel em:

http://www.scielo.br/scielo.php?script=sci_arttext\&pid=S0100-

69162005000200003\&lng=pt\&nrm=iso. Acesso em: 07 ago. 2010.

PERISSINOTTO, M.; MOURA, D.J.; CRUZ, V.F.; SOUZA, S.R.L.; LIMA, K.A.O.; MENDES, A.S. Conforto térmico de bovinos leiteiros confinados em clima subtropical e mediterrâneo pela análise de parâmetros fisiológicos, utilizando a teoria dos conjuntos fuzzy. Ciência Rural, Santa Maria, v.39, n.5, p.1.492-1.498, 2009.

ROUSING, T.; WEMELSFELDER, F. Qualitative assessment of social behaviour of dairy cows housed in loose housing systems. Applied Animal Behaviour Science, Bristol, v.101, p.40-53, 2006.

SARIEGO, J.C.L. O que é Etologia? Online Disponivel em:

http://paginas.terra.com.br/educacao/sariego/o_estudo_do_comportamento.htm. Acesso em 29 abr. 2005.

SCHUTZ, K.E.; ROGERS, A.R.; COX, N.R.; TUCKER, C.B. Dairy cows prefer shade that offers greater protection against solar radiation in summer: Shade use, behaviour, and body temperature. Applied Animal Behaviour Science, Bristol, v.116, p.28-34, 2009.

SCHWAGER, M.; ANDERSON, D.M.; BUTLER, Z.; RUS, D. Robust classification of animal tracking data. Computers and Electronics in Agriculture, Amsterdam, v.56, n.1, p.46-59, 2007.

TEIXEIRA, D.L.; HÖTZEL, M.J.; MACHADO FILHO, L.C. Designing better water troughs 2. Surface area and height, but not depth, influence dairy cows' preference. Applied Animal Behaviour Science, Bristol, v.96, n 1-2, p.169-175, 2006.

TREVISAN, N.B.; QUADROS, F.L.F.; SILVA, A.C.F.; BANDINELLI, D.G.; MARTINS, C.E.N.; SIMÕES, L.F.C.; MAIXNER, A.R.; PIRES, D.R.F. Comportamento ingestivo de novilhos de corte em pastagem de aveia-preta e azevém com níveis distintos de folhas verdes. Ciência Rural, Santa Maria, v. 34, n.5, p.1.543-1.548, 2004. 